\title{
Coronavirus (SARS-CoV-2) y el entorno odontológico.
}

\author{
Coronavirus (SARS-CoV-2) and the dental environment.
}

\author{
José Eduardo Orellana-Centeno,* Verónica Morales-Castillo, ${ }^{\ddagger}$ Roxana Nayeli Guerrero Sotelo ${ }^{\S}$
}

\section{RESUMEN}

El coronavirus (SARS-CoV-2) es un virus que afecta las vías respiratorias del huésped infectado, provocando daños no solamente a ese nivel, sino una disfunción multiorgánica que provoca la muerte de la persona infectada. Factores como la edad (adultos mayores), comorbilidades (obesidad, diabetes, hipertensión, etcétera) favorecen que la afectación por este virus sea más letal. Esta pandemia que inició en China y se ha expandido en una gran cantidad de países en todo el mundo ha obligado a los servicios de salud no solamente a atender la pandemia, sino a desarrollar protocolos para la atención de los pacientes y la protección del personal de salud.

Palabras clave: COVID-19, coronavirus, control de infección, odontología.

\section{ABSTRACT}

The coronavirus (SARS-CoV-2) is a virus that affects the airways of the infected host, causing damage not only at that level, but also multiorgan dysfunction that causes its death. Factors such as age (older adults), comorbidities (obesity, diabetes, hypertension, etc.) favor that the affectation by this virus is more lethal. This pandemic that started in China and has spread in many countries around the world has forced health services not only to attend to the pandemic but also to develop protocols for the care of patients for their protection and the personnel of health.

Keywords: COVID-19, coronavirus, infection control, odontology.

\section{INTRODUCCIÓN}

E n diciembre de 2019 en Wuhan, ciudad capital de la provincia de Hubei, se presentaron los primeros casos de neumonía severa con etiología desconocida o por consumo de alimentos en cierto mercado de la zona especialista en animales vivos exóticos. El 31 de diciembre del 2019 la Organización Mundial de la Salud fue notificada de la presencia de esta enfermedad, unos días después el virus fue identificado como coronavirus, que tenía una relación con el coronavirus del murciélago (> 95\%) y similar al SARS CoV (> 70\%). ${ }^{1}$ Aunque el SARS-CoV-2 se originó a partir de los murciélagos, el animal intermediario a través del cual se transfirió es incierto. Otros animales como son los pangolines y las serpientes son sospechosos. Inició un aumento de casos exponencialmente, lo que sugiere que la transmisión humano-humano había iniciado, ya que no eran casos con antecedentes de consumo o laborar en dicho mercado. Adicionalmente, la migración masiva aumentó la epidemia no solamente en otras provincias de China, sino que también algunos países de Asia (Tailandia, Japón y Corea del Sur). Hubo la necesidad de restringir la entrada y salida en la región de Wuhan. ${ }^{2}$

Algunos aeropuertos en el mundo comenzaron a instaurar mecanismos de detección para identificar personas sintomáticas y limitar el ingreso a los países con la finalidad de blindarse contra el virus. Otra de las acciones realizadas

\footnotetext{
* Profesor Investigador de tiempo completo (Maestro en Salud Pública). Universidad de la Sierra Sur, Instituto de Investigación Sobre la Salud Pública, Licenciatura de Odontología. Oaxaca, México.

₹ Médico Especialista en Medicina Familiar. Unidad Académica Multidisciplinaria Zona Media Universidad Autónoma de San Luis Potosí, Maestría en Administración. San Luis Potosí, México.

§ Profesor Investigador de tiempo completo (Doctora). Universidad de la Sierra Sur, Instituto de Investigación Sobre la Salud Pública, Licenciatura de Enfermería. Oaxaca, México.
} 
fue iniciar la evacuación de sus ciudadanos provenientes desde Wuhan por medio de vuelos especiales, colocando a todas las personas sintomáticas de forma aislada durante catorce días. $^{3}$

El 30 de enero, el Comité de Emergencias de la Organización Mundial de la Salud (OMS) declaró al brote de COVID-19 como una «emergencia de salud pública de importancia internacional», lo que significa que los países del mundo deben prepararse para la detección oportuna de casos importados y posteriormente, los brotes de transmisión autóctona. Con ello se inició la implementación de los mecanismos de respuesta a lo que pudiera ser una pandemia. ${ }^{4}$

Los estudios informaron de un tiempo de duplicación de los casos en sólo ocho días. En febrero, China cambió su definición de casos confirmados para incluir pacientes con negativo, que son aquéllos que están pendientes de pruebas moleculares. Llegó a presentarse un aumento de casos por 15 mil en un solo día. A partir de marzo, se presentaron 96 mil casos en todo el mundo, cerca de 80 mil casos solamente en China, y presentes en 87 países. $^{5}$

A partir de abril, inició la disminución de casos en China, pero aumentó exponencialmente en otros países como Corea del Sur, Italia, España y Estados Unidos como se puede ver en la Tabla 1.

Es una infección que se transmite a través de gotas generadas al estornudar y toser en personas asintomáticas, antes del inicio de los síntomas o sintomáticos. Los pacientes pueden ser infecciosos mientras los síntomas estén presentes. Estas gotas, provenientes de personas infectadas, pueden extenderse de uno a dos metros y permanecer durante cierto tiempo en múltiples superficies. El entrar en contacto con estas superficies contaminadas y después de ello tocarse la nariz, boca y ojos. ${ }^{6}$

El virus puede destruirse en menos de un minuto con desinfectantes comunes como el hipoclorito de sodio, peróxido de hidrogeno, agua y jabón, etcétera.

\section{CARACTERÍSTICAS CLÍNICAS}

Se presenta desde un estado asintomático al síndrome agudo respiratorio y disfunción multiorgánica. Dentro de los síntomas se incluyen: tos, dolor de garganta, cabeza, fatiga, mialgia, conjuntivitis y disnea. Es indistinguible de otras infecciones respiratorias similares, y al final de la primera semana puede progresar a neumonía respiratoria y muerte. ${ }^{7}$

La media desde el inicio de los síntomas hasta la disnea fue de cinco días y hospitalización a partir del día siete. La necesidad de ingreso en cuidados intensivos está entre el $25-30 \%$ de los pacientes afectados. Dentro de las complicaciones se incluye lesión pulmonar aguda, shock y lesión renal. La duración de la estancia hospitalaria es de aproximadamente 10 días y la recuperación de los pacientes es entre dos o tres semanas aproximadamente. ${ }^{8}$

Algunos factores por considerar son la edad, siendo más común los adultos mayores en presentar dificultades y posterior deceso, así como también aquéllos con comorbilidades (50-75\% casos fatales). Por el momento no existe un tratamiento específico ni tampoco se cuenta con vacuna. ${ }^{9}$

\section{DIAGNÓSTICO}

Los casos sospechosos son aquéllos que presentan tos, fiebre superior a los $38{ }^{\circ} \mathrm{C}$ y dolor de garganta, en un principio con antecedentes de viaje al extranjero, esto último ya no se considera como un elemento necesario para el diagnóstico. Se debe considerar que los casos pueden ser asintomáticos o no presentar fiebre. Solamente puede considerarse un caso confirmado hasta que es positivo en la prueba de biología molecular. Dichas pruebas deben realizarse en laboratorios autorizados por la Secretaría de Salud, en una fase inicial solamente eran laboratorios estatales, poco a poco se incorporaron algunos laboratorios de universidades públicas y algunas instituciones privadas. ${ }^{10}$

En cuanto a algunas otras pruebas de laboratorio, cabe mencionar que puede haber linfopenia (recuento de linfocitos $<1,000$ ), recuento de plaquetas normal o ligeramente bajo. Niveles de procalcitonina normales y si es ligeramente alta, indica coinfección bacteriana.

\section{Tabla 1: Pandemia de COVID-19 a 50 días por país.}

\begin{tabular}{lcc} 
País & Número de casos & $\begin{array}{c}\text { Número de } \\
\text { decesos }\end{array}$ \\
\hline México & 754 & 26 \\
Estados Unidos & 5,877 & 449 \\
$\quad$ de América & \\
Ecuador & 7,858 & $388^{*}$ \\
Corea del Sur & 7,382 & 51 \\
Italia & 41,035 & 3,407 \\
España & 19,980 & 1,002 \\
\hline * Apenas cumple 48 días al momento de la elaboración. \\
Fuente: Elaboración propia.
\end{tabular}


Niveles altos de AST/ALT (aminotransferasa y alanino aminotransferasa), protrombina, creatinina y LDH (lactate dehydrogenase) están asociados con enfermedad grave. La radiografía de tórax generalmente presenta infiltrados bilaterales en la etapa temprana. La tomografía computarizada muestra infiltrados, opacidades en vidrio esmerilado y consolidación subsegmentaria. ${ }^{11}$

El diagnóstico diferencial incluye enfermedades infecciosas virales en vías respiratorias como influenza, parainfluenza, adenovirus, etcétera, e infecciones bacterianas. No se puede descartar de manera clínica o a través de pruebas de laboratorio de rutina.

\section{TRATAMIENTO}

El tratamiento es esencialmente de soporte y sintomático. El primer paso es aislamiento para evitar la transmisión a otras personas (medida aplicada en México por medio de la Jornada Nacional de «Susana distancia»). La enfermedad leve debe ser manejada en casa, manteniéndose bien alimentados, hidratados y controlando la fiebre, debe evitarse el uso de antibióticos y antivirales. En pacientes con complicaciones se requiere hospitalización y su traslado debe hacerse con todo cuidado de acuerdo con los protocolos establecidos. En pacientes hipóxicos suministrarles oxígeno a través de mascarilla, cánula nasal de alto flujo o ventilación no invasiva, y en caso de que se requiera, la ventilación mecánica. ${ }^{12}$

\section{PREVENCIÓN}

En este momento no hay tratamientos aprobados, la prevención se considera crucial el aislamiento de casos confirmados o sospechosos y permanecer en casa. Se les debe pedir a los casos sospechosos el uso de mascara quirúrgica simple (cubrebocas) y practicar el estornudo de «etiqueta» (estornudar con el ángulo interno del antebrazo). Maximizar los niveles de higiene en casa, trabajo y zonas comunes, así como también lavado de manos con agua y jabón (mínimo 20 segundos) y el uso de gel antibacterial. A nivel comunitario, se recomienda no asistir a lugares concurridos, evitar la asistencia escolar y disminuir al mínimo la apertura de comercios y otras industrias no indispensables, así como evitar el traslado y posponer viajes no esenciales, sobre todo a lugares con casos confirmados. ${ }^{13}$

Las recomendaciones para el personal de salud son limpiar y sanitizar las superficies del equipo, instrumental e infraestructura preferiblemente con hipoclorito de sodio. Los médicos y trabajadores de la salud deben estar provistos de respiradores tipo N95 preferentemente, uso de gafas o mascara para protección, batas desechables gruesas que sean impermeables y extremar los cuidados para evitar contagio. ${ }^{14}$

\section{COVID-19 Y EL ENTORNO ODONTOLÓGICO}

La clínica o consultorio odontológico no están exentos de evitar adquirir o transmitir infecciones entre las personas. De no seguir medidas de bioseguridad, un consultorio podría ser un ambiente riesgoso para la propagación de virus debido al contacto cercano con los pacientes. Aunque los pacientes con diagnóstico de COVID-19 deben realizarse solamente tratamientos dentales por una emergencia. Una de las mayores preocupaciones para los odontólogos sin duda son los pacientes infectados que no muestran síntomas, por lo tanto se debe ser muy cuidadoso al tratar pacientes asintomáticos y tratar a todos manteniendo las medidas de bioseguridad. ${ }^{15}$

Existen pautas prácticas recomendadas al personal de salud por el Centro de Control de Enfermedades (CDC), la Asociación Dental Americana (ADA) y la Organización Mundial de la Salud (OMS) para controlar la propagación del virus. Estas recomendaciones incluyen equipo de protección, lavado de manos, evaluación detallada del paciente, aislamiento con dique de hule y enjuague bucal antes de cualquier procedimiento dental. ${ }^{16-18}$

\section{BIBLIOGRAFÍA}

1. Huang C, Wang Y, Li X et al. Clinical features of patients infected with 2019 novel coronavirus in Wuhan, China. Lancet. 2020; 395: 497-506.

2. Wang C, Horby PW, Hayden FG, Gao GF. A novel coronavirus outbreak of global health concern. Lancet. 2020; 395 (10223): 470-473. Disponible en: https://doi.org/10.1016/S0140-6736(20)30185-9.

3. World Health Organization. Situation reports. Disponible en: https:// www.who.int/emergencies/diseases/novel-coronavirus-2019/ situation-reports/

4. World Health Organization. Coronavirus disease (COVID-19) outbreak. Ginebra: WHO; 2020. Disponible en: https://www.who. int/emergencies/diseases/novel-coronavirus-2019

5. Li Q, Guan X, Wu P et al. Early transmission dynamics in Wuhan, China, of novel coronavirus-infected pneumonia. N Engl J Med. 2020; 382 (13): 1199-1207. Disponible en: https://doi. org/10.1056/NEJMoa2001316.

6. Zou L, Ruan F, Huang M et al. SARS-CoV-2 viral load in upper respiratory specimens of infected patients. N Engl J Med. 2020; 382 (12): 1177-1179. Disponible en_https://doi.org/10.1056/ NEJMc2001737.

7. Chen N, Zhou M, Dong X et al. Epidemiological and clinical characteristics of 99 cases of 2019 novel coronavirus pneumonia in Wuhan, China: a descriptive study. Lancet. 2020; 395: 507-513.

8. Xu XW, Wu XX, Jiang XG et al. Clinical findings in a group of patients infected with the 2019 novel coronavirus (SARS-CoV-2) outside of Wuhan, China: retrospective case series. BMJ. 2020; 368: m606. 
9. Na Z, Zhang D, Wang W, Xingwang L, Bo Y, Jingdong S et al. A novel coronavirus from patients with pneumonia in China, 2019. Engl J Med. 2020; 382: 727-733.

10. Jin $\mathrm{YH}$, Cai $\mathrm{L}$, Cheng $\mathrm{ZS}$ et al. A rapid advice guideline for the diagnosis and treatment of 2019 novel coronavirus (2019-nCoV) infected pneumonia. Mil Med Res. 2020; 7: 4.

11. Huang P, Liu T, Huang $L$ et al. Use of chest CT in combination with negative RT-PCR assay for the 2019 novel coronavirus but high clinical suspicion. Radiology. 2020; 295 (1): 22-23. Disponible en: https://doi.org/10.1148/radiol.2020200330.

12. WHO. Clinical management of severe acute respiratory infection when novel coronavirus [nCoV] infection is suspected. Disponible en: https://www.who.int/publications-detail/clinicalmanagementof- severe-acute-respiratory-infection-whennovelcoronavirus-[ncov]-infection-is-suspected.

13. World Health Organization. Coronavirus disease (COVID-19). Technical guidance: infection prevention and control. Disponible en: https://www.who.int/emergencies/diseases/novelcoronavirus-2019/technical-guidance/infection-prevention-andcontrol.

14. Chang D, Xu H, Rebaza A, Sharma L, De la Cruz CS. Protecting health-care workers from subclinical coronavirus infection. Lancet Respir Med. 2020; 8 (3): e13. Disponible en: https://doi. org/10.1016/S2213-2600(20)30066-7.

15. Meng L, Hua F, Bian Z. Coronavirus disease 2019 (COVID-19): emerging and future challenges for dental and oral medicine. J Dent Res. 2020; 99 (5): 481-487. doi: 10.1177/0022034520914246.
16. The World Health Organization (WHO). Clinical management of severe acute respiratory infection when novel coronavirus (nCoV) infection is suspected: Interim guidance. 2020. Disponible en: https://www.who.int/publications-detail/clinical-managementofsevere-acute-respiratory-infection-when-novel-coronavirus(ncov)-infection-is-suspected.

17. Centers for Disease Control and Prevention (CDC). CDC Developing Guidance Regarding Responding to COVID-19 in Dental Settings. Division of Oral Health, National Center for Chronic Disease Prevention and Health Promotion; 2020. Disponible en: https:// www.cdc.gov/oralhealth/infectioncontrol/statement-COVID.html.

18. The American Dental Association (ADA). Coronavirus frequently asked questions. 2020. Disponible en: https://success.ada.org/ en/practice-management/patients/coronavirus-frequently-askedquestions.

\section{Correspondencia:}

\section{José Eduardo Orellana-Centeno}

Universidad de la Sierra Sur.

Guillermo Rojas Mijangos s/n, esq. Av. Universidad, 70800, Miahuatlán de Porfirio Díaz,

Oaxaca, México.

E-mail: orellana17@msn.com 